\title{
Solving system of nonlinear integral equations by Newton-Kantorovich method
}

\begin{abstract}
Newton-Kantorovich method is applied to obtain an approximate solution for a system of nonlinear Volterra integral equations which describes a large class of problems in ecology, economics, medicine and other fields. The system of nonlinear integral equations is reduced to find the roots of nonlinear integral operator. This nonlinear integral operator is solved by the Newton-Kantorovich method with initial guess and this procedure is continued by iteration method to find the unknown functions. Finally, numerical examples are provided to show the validity and the efficiency of the method presented.
\end{abstract}

Keyword: Newton-Kantorovich method; Nonlinear operator; Volterra integral equation 